International Journal of Pure and Applied Mathematics

Volume 110 No. 2 2016, 383-387

ISSN: 1311-8080 (printed version); ISSN: 1314-3395 (on-line version)

url: http://www.ijpam.eu

doi: 10.12732/ijpam.v110i2.12

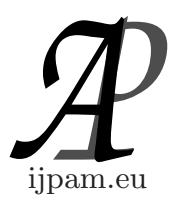

\title{
ON FUNCTIONS WITH STRONGLY CLOSED GRAPH
}

\author{
Asha Gupta ${ }^{1}$, Kamal Kishore ${ }^{2}$ \\ ${ }^{1,2}$ Department of Applied Sciences \\ PEC University of Technology \\ Chandigarh, INDIA
}

\begin{abstract}
The primary purpose of this paper is to study functions with strongly closed graph and consequently a characterization of such functions in terms of r-convergence of nets is obtained. Further, conditions are investigated under which other functions such as continuous, almost continuous, closed, almost closed functions have strongly closed graph.
\end{abstract}

AMS Subject Classification: 54A20, 54C10

Key Words: Continuous, closed, strongly closed graph.

\section{Introduction}

By a space, we shall mean a topological space. No separation axioms are assumed and no function is assumed to be continuous or onto unless mentioned explicitly; $\operatorname{cl}(A)$ will denote the closure of the subset $A$ in the space $X$. We will denote nets by symbol such as $x_{\alpha}$. A subnet of $x_{\alpha}$ will be denoted by $x_{N b}$ where $b$ is a member of the domain of $x_{N b}$ and $N$ is the appropriate function from the domain of $x_{N b}$ to the domain of $x_{\alpha}$. We say that the net $x_{\alpha}$ r-converges to $x\left(x_{\alpha} \rightarrow_{r} x\right)$ if for every open set $U$ containing $x$ there exists a $\alpha_{0}$ such that $x_{\alpha} \in \operatorname{cl} U$ for all $\alpha \geq \alpha_{0}$.

Received: $\quad$ June 24, 2016

Revised: October 21, 2016

Published: November 4, 2016

$\S_{\text {Correspondence author }}$ (c) 2016 Academic Publications, Ltd. url: www.acadpubl.eu 
A function $f: X \rightarrow Y$ is said to be almost continuous [2] if $f^{-1}(V)$ is open in $X$ for every regular open subset $V$ of $Y$ or equivalently, $f^{-1}(F)$ is closed in $X$ for every regular closed subset $F$ of $Y$. A space $X$ is said to be H-closed [2] if for every open cover $\left\{U_{\alpha}: \alpha \in \Delta\right\}$ there exists a finite subfamily $\left\{U_{\alpha i}: i=1,2, \ldots, n\right\}$ such that $\cup\left\{\operatorname{cl}\left(U_{\alpha i}\right): 1 \leq i \leq n\right\}=X$. If $f: X \rightarrow Y$ is a function, then fibers are the sets $f^{-1}(y)$ where $y \in Y . f$ is said to have closed graph if graph of $f$, that is, the set $\{(x, f(x)): x \in X\}$ is a closed subset of the product space $X \times Y$. $f$ is said to have strongly closed graph [3] if $x \in X$, $y \in Y, y \neq f(x)$ implies there exist open sets $U$ and $V$ containing $x$ and $y$ respectively such that $f(U) \cap c l V=\phi$.

P.E. Long [5] has obtained the following characterization of functions with closed graph.

Theorem 1.1. A function $f: X \rightarrow Y$ has closed graph if $x \in X, y \in Y$, $y \neq f(x)$ implies there exist open sets $U$ and $V$ containing $x$ and $y$ respectively such that $f(U) \cap V=\phi$.

Remark 1.2. Any function with strongly closed graph also has closed graph.

\section{Main Results}

Using the characterizations of closed sets, Kelley [4,chapter 2] has given following characterization of functions with closed graph in terms of nets.

Theorem 2.1. A function $f: X \rightarrow Y$ has closed graph if and only if $x_{\alpha} \rightarrow x$ in $X$ and $f\left(x_{\alpha}\right) \rightarrow y$ in $Y$ implies $y=f(x)$.

The following theorem which is an analogue of the above theorem gives a characterization of functions with strongly closed graph in terms of r-convergence of nets.

Theorem 2.2. Let $f: X \rightarrow Y$ be any function, where $Y$ is regular. Then the following are equivalent:

(a) $f$ has strongly closed graph.

(b) $x_{\alpha} \rightarrow x$ in $X$ and $f\left(x_{\alpha}\right) \rightarrow_{r} y$ in $Y$ implies $y=f(x)$.

(c) $x_{\alpha} \rightarrow x$ in $X$ and $f\left(x_{\alpha}\right) \rightarrow y$ in $Y$ implies $y=f(x)$.

(d) $f$ has closed graph.

Proof. $(a) \Rightarrow(b)$ Suppose (a) holds. Let $x_{\alpha} \rightarrow x$ in $X$ and $f\left(x_{\alpha}\right) \rightarrow_{r} y$ in $Y$. To prove $y=f(x)$. Suppose $y \neq f(x)$. Then $f$ has strongly closed graph 
implies there exist open sets $U$ and $V$ containing $x$ and $y$ respectively such that $f(U) \cap c l V=\phi$. Now $x_{\alpha} \rightarrow x$ implies there exists $\alpha_{0}$ such that $x_{\alpha} \in U$ for all $\alpha \geq \alpha_{0}$ and $f\left(x_{\alpha}\right) \rightarrow_{r} y$ implies there exists $\alpha_{1}$ such that $f\left(x_{\alpha}\right) \in c l V$ for all $\alpha \geq \alpha_{1}$. Let $\alpha_{2}=\max \left(\alpha_{0}, \alpha_{1}\right)$. Then $f\left(x_{\alpha}\right) \in f(U) \cap c l V$ for all $\alpha \geq \alpha_{2}$ i.e. $f(U) \cap c l V \neq \phi$-a contradiction. Hence (b) holds.

$(b) \Rightarrow(c)$ is obvious.

$(c) \Rightarrow(d)$ follows from the above theorem 2.1.

$(d) \Rightarrow(a)$ Suppose (d) holds. To prove $f$ has strongly closed graph , let $y \neq f(x)$. Then $f$ has closed graph implies there exist open sets $U$ and $V$ containing $x$ and $y$ respectively such that $f(U) \cap V=\phi$. Since $Y$ is regular, there exists an open set $W$ containing $y$ such that $c l W \subset V$ implying thereby that $f(U) \cap c l W=\phi$. Hence $f$ has strongly closed graph.

We now turn to gathering some more facts about the functions with strongly closed graph and their relations to other functions. Fuller has proved that a continuous function $f: X \rightarrow Y$ has closed graph if $Y$ is Hausdorff [1, Theorem 3.4]. We give more stronger result by proving that even an almost continuous function has strongly closed graph if $Y$ is Hausdorff, the proof of which is as follows.

Theorem 2.3. Let $f: X \rightarrow Y$ be almost continuous function where $Y$ is Hausdorff. Then $f$ has strongly closed graph.

Proof. Let $x \in X, y \in Y, y \neq f(x)$. Then $Y$ is Hausdorff implies there exist open sets $V$ and $W$ containing $f(x)$ and $y$ respectively such that $V \cap W=\phi$. This implies $V \cap c l W=\phi$. Since $f$ is almost continuous $f^{-1}(c l W)$ is closed and $x \notin f^{-1}(c l W)$. Let $U=X-f^{-1}(c l W)$. Then $U$ is an open set containing $x$ and $f(U) \cap c l W=\phi$ implying thereby that $f$ has strongly closed graph.

Corollary 2.4. Let $f: X \rightarrow Y$ be continuous function where $Y$ is Hausdorff. Then $f$ has strongly closed graph.

A function with strongly closed graph need not be almost continuous [3,Example 2]. Further a function with closed graph takes compact subsets of $Y$ onto closed subsets of $X$ [1,Theorem 3.6] and the map becomes continuous if $Y$ is compact. We now provide parallel results for functions with strongly closed graph.

Lemma 2.5. Let $f: X \rightarrow Y$ be a function with strongly closed graph. Then inverse image of each $H$-closed subset of $Y$ is closed.

Proof. Let $A$ be an H-closed subset of $Y$ and let $x \in c l f^{-1}(A)$. This implies there exists a net $x_{\alpha}$ in $f^{-1}(A)$ such that $x_{\alpha} \rightarrow x$. Now $f\left(x_{\alpha}\right)$ being a net 
in H-closed set $A$ has a subnet $f\left(x_{N b}\right)$ which r-converges to $y$ in $A$. Now $x_{N b}$ being a subnet of $x_{\alpha}$ converges to $x, f\left(x_{N b}\right)$ r-converges to $y$ and $f$ has strongly closed graph implies $y=f(x)$. Therefore $x \in f^{-1}(A)$. Hence $f^{-1}(A)$ is closed.

Theorem 2.6. Let $f: X \rightarrow Y$ be a function with strongly closed graph where $Y$ is $H$-closed. Then $f$ is almost continuous.

Proof. The proof follows from above lemma and the fact that a regular closed subset of H-closed space is H-closed.

Remark 2.7. Example 3 of [3] shows that the strong closed graph condition in theorem 2.6 can not be relaxed to closed graph condition.

Combining Theorem 2.3 and theorem 2.6, we get the following theorem.

Theorem 2.8. Let $f: X \rightarrow Y$ be a function where $Y$ is Hausdorff and $H$-closed. Then $f$ is almost continuous if and only if $f$ has strongly closed graph.

Having already discovered the conditions under which an almost continuous or continuous function has strongly closed graph, we proceed to find conditions under which an almost closed or closed function has strongly closed graph. Fuller has proved that a closed function $f: X \rightarrow Y$ with closed fibers has closed graph if $X$ is regular[1,corollary 3.9], we give the following result (nonparenthesis part) by proving that even an almost closed function has strongly closed graph, while the parenthesis part is analogue of the non-parenthesis part.

Theorem 2.9. Let $f: X \rightarrow Y$ be almost closed with closed(compact) fibers where $X$ is regular(Hausdorff) and $Y$ is regular, then $f$ has strongly closed graph.

Proof. We prove the parenthesis part, the proof of the non parenthesis part is similar. Let $x \in X, y \in Y, y \neq f(x)$.Then $x \notin f^{-1}(y)$ which is compact, as $f$ has compact fibers. Since $X$ is Hausdorff, there exist open sets $U$ and $V$ containing $x$ and $f^{-1}(y)$ such that $U \cap V=\phi$. This implies $c l U \cap V=\phi$. As $f$ is almost closed, $f(c l U)$ is closed and $y \notin f(c l U)$. Let $W=Y-f(c l U)$. Then $W$ is an open set containing $y$ and $f(c l U) \cap W=\phi$ or $f(U) \cap W=\phi$. Since $Y$ is regular, there exists an open set $W^{\prime}$ containing $y$ such that $c l W^{\prime} \subset W$. Therefore $f(U) \cap c l W^{\prime}=\phi$ implying thereby that $f$ has strongly closed graph.

Corollary 2.10. Let $f: X \rightarrow Y$ be closed with closed (compact) fibers where $X$ is regular (Hausdorff) and $Y$ is regular, then $f$ has strongly closed graph. 


\section{References}

[1] R. V. Fuller, Relations among continuous and various non continuous functions, Pacific J. Math., 25 (1968), 495-509.

[2] T.R. Hamlett, L.L. Herrington, The closed graph and p-closed properties in general topology, Amer. Math. Soc., Providence Rhode Island (1981).

[3] L.L. Herrington, P.E. Long, Characterizations Of H-closed spaces, Proc. Amer. Math. Soc., 48, No. 2 (1975), 468-475.

[4] J.L. Kelly, General Topology, D. Van Nostrand, Co. Princeton (1955).

[5] P.E. Long, Functions with closed graph, Amer. Math. Monthly, 16, No. 8 (1969), 930-932. 
